\title{
Challenges in the description of the atomic nucleus: Unification and interdisciplinarity
}

\author{
P.F. Bortignon ${ }^{1,2, a}$ and R.A. Broglia ${ }^{1,3}$ \\ 1 Department of Physics, University of Milan, Milan, Italy \\ 2 INFN, Sezione Milano, Milan, Italy \\ 3 The Niels Bohr Institute, University of Copenhagen, Copenhagen, Denmark
}

Received: 19 February 2016

Published online: 23 March 2016

(c) The Author(s) 2016. This article is published with open access at Springerlink.com Communicated by N. Alamanos

\begin{abstract}
Nuclear physics, in general, and theoretical nuclear physics, in particular, have provided the physics community at large, among other things, with the paradigm of spontaneous symmetry breaking phenomena in finite many-body systems. The study of the associated mechanisms of symmetry restoration has shed light on the microscopic structure of the corresponding condensates, in particular on the superfluid phase, allowing to study Cooper pair tunnelling into superfluid nuclei (related to the Josephson effect), in terms of individual quantum states and reaching, in doing so, a new milestone: that of unifying structure and reactions, these last processes being found at the basis of the formulation of quantum mechanics (probability interpretation, Born). In the process, nuclear physicists have extended the validity of BCS theory of superconductivity to the single Cooper pair situation, let alone discovering unexpected mechanism to break gauge invariance. The insight obtained from pair transfer research is likely to have important consequences in the study of double charge exchange processes, and thus in the determination of the nuclear matrix element associated with neutrinoless double beta decay, eventually providing an important test of the Standard Model. Time, thus, seems ripe for nuclear theorists to take centre stage, backed by a wealth of experimental information and by their interdisciplinary capacity to connect basic physical concepts across the borders. With the help of these elements they can aim at fully revealing the many facets of their femtometer many-body system, from vacuum zero point fluctuations to new exotic modes of nuclear excitations and of their interweaving, resulting in powerful effective field theories. Unless. Unless they are not able to free themselves from words like $a b$ initio or fundamental, and to adapt a relax attitude concerning Skyrme, tensor, etc., forces, as well as regarding the quest for "the" Hamiltonian.
\end{abstract}

The modern theory of nuclear structure results from the merging of the liquid drop and of the shell model, which contributed to the concepts of collective excitations and of independent-particle motion, respectively. These apparently contradictory views became eventually unified in the paradigm of broken symmetry restoration to determine the elementary modes of nuclear excitation, their interweaving being a consequence of the particle-vibration coupling mechanism [1]. The resulting clothed bosonic and fermionic degrees of freedom constitute the physical, elementary modes of nuclear excitation which diagonalize the many-body nuclear Hamiltonian restoring symmetries spontaneously broken. The associated spectroscopic amplitudes provide, together with the theory of reactions [2$5]$, the elements to calculate the absolute value of the differential cross sections and transition rates. In partic-

\footnotetext{
${ }^{a}$ e-mail: Pierfrancesco.Bortignon@mi.infn.it
}

ular, those associated with: a) inelastic scattering and Coulomb excitation, b) one-particle and, c) two-particle transfer processes. The corresponding spectroscopic amplitudes and associated formfactors also provide the input to work out the optical potentials needed in the calculation of the absolute cross sections, quantities which can be compared directly with the experimental data.

The above elements testify the need for a new level of unification of the variety of facets of theoretical nuclear physics: that between structure and reactions, let alone that between the physics of bound and continuum states. This unification, still in the making, will prove essential to meet the challenges resulting from experimental developments and, also, for those coming from other areas of physics, that is, from interdisciplinary research.

The importance of talk across the borders can hardly be overemphasized. Without the input from condensed matter, the theory of nuclear pairing as we know it today would not be $[6,1,7]$, nor the description of two-nucleon 
transfer reactions, mainly as a successive transfer [8-10], which can be calculated with high accuracy, rendering quantitative the probing of pairing in nuclei [11].

Let us now go back to a), b) and c). Processes a) and c), specifically probe collective modes. The first ones are those associated with correlated particle-hole $(p h)$ states, the second ones correspond to correlated $(p p)$ and $(h h)$ states, that is, pairing vibrations; in particular, the newly discovered Giant Pairing Vibrations (GPV) [12,13], in the two-nucleon transfer reactions ${ }^{12} \mathrm{C}\left({ }^{18} \mathrm{O},{ }^{16} \mathrm{O}\right){ }^{14} \mathrm{C}$ and ${ }^{13} \mathrm{C}\left({ }^{18} \mathrm{O},{ }^{16} \mathrm{O}\right){ }^{15} \mathrm{C}$.

Reactions of type b) give specific information concerning the single-particle content of nuclear states [14] as well as those lying in the continuum as testified by the analysis [15] of the reaction ${ }^{9} \mathrm{Li}(d, p){ }^{10} \mathrm{Li}[16]$ populating the parity inverted, virtual $s_{1 / 2}$ and resonant $p_{1 / 2}$ valence states lying at threshold, and responsible for the new magic number $N=6$ and for many of the exotic properties of the nucleus ${ }^{11} \mathrm{Li}[17,18]$. In particular, the fact that most of the pairing energy with which the neutron halo Cooper pair binds to the ${ }^{9} \mathrm{Li}$ core, is due to the exchange of the low-energy E1-mode of ${ }^{11} \mathrm{Li}$ (which can be viewed as the paradigm of dipole vibrations in presence of an overwhelming neutron skin, namely the scenario of the Giant Dipole Pigmy Resonance (GPDR)), and of the low-lying quadrupole vibration of ${ }^{8} \mathrm{He}$, as testified by the absolute value of the differential cross section associated with the states populated in the reaction ${ }^{1} \mathrm{H}\left({ }^{11} \mathrm{Li},{ }^{9} \mathrm{Li}\right){ }^{3} \mathrm{H}[19,20]$.

New frontiers of interdisciplinarity and unification are being forced open by work on double charge-exchange $(2 n, 2 p)$ and neutrinoless double beta decay $(\beta \beta(0 \nu))$, this last constituting an important element in testing the Standard Model. Nuclear matrix elements entering the calculation of $\beta \beta(0 \nu)$ can be, in principle, extracted from the absolute value of $(2 n, 2 p)$ differential cross sections [21-25], in particular, of the ${ }^{40} \mathrm{Ca}\left({ }^{18} \mathrm{O},{ }^{18} \mathrm{Ne}\right){ }^{40} \mathrm{Ar}$ process [26]. A quantitative control of the accuracy with which one is able to calculate the elements entering $\mathrm{d} \sigma\left(\theta ;{ }^{40} \mathrm{Ca} \rightarrow{ }^{40} \mathrm{Ar}\right) / \mathrm{d} \Omega$, the absolute value of the single charge-exchange process ${ }^{40} \mathrm{Ca}\left({ }^{18} \mathrm{O},{ }^{18} \mathrm{~F}\right){ }^{40} \mathrm{~K}$ and of the two-neutron and two-proton transfer reactions, ${ }^{40} \mathrm{Ca}\left({ }^{18} \mathrm{O},{ }^{16} \mathrm{O}\right){ }^{42} \mathrm{Ca}$ and ${ }^{40} \mathrm{C}\left({ }^{18} \mathrm{O},{ }^{20} \mathrm{Ne}\right){ }^{38} \mathrm{Ar}$, have to be calculated, and the results compared with the experimental findings. In this way the three channels contributing to the ${ }^{40} \mathrm{Ca} \rightarrow{ }^{40} \mathrm{Ar}$ reaction will be properly characterized, in terms of GT wave functions for the first one, and of pairing vibrations for the last two. With a number of provisos, however. First, all these vibrations have to be renormalized in terms of self-energy and vertex corrections arising from the coupling to low-lying collective $(p h)$ excitations [27], and through the mixing of particle and hole states in the case in which the collective mode is a pairing vibration $[28,29]$. Second, within the context of double charge exchange, both GT and pairing vibrations have to be extended to other multipolarities different from $1^{+}$and $0^{+}$, respectively. Last, but not least, the ground state of both ${ }^{16} \mathrm{O}$ and ${ }^{40} \mathrm{Ca}$ contain $n p-n h$ (coexistent) deformed components. These components have to be properly dealt with in the quest for a quantitative description of the ${ }^{40} \mathrm{Ca} \rightarrow{ }^{40} \mathrm{Ar}$ process. The need for a broad interdisciplinary background becomes apparent. Further insight concerning how to quantitatively deal with the GT modes could be provided by a study of the reactions ${ }^{96} \mathrm{Mo}\left(d,{ }^{2} \mathrm{He}\right)^{96} \mathrm{Nb}$ and ${ }^{96} \mathrm{Zr}\left({ }^{3} \mathrm{He}, t\right){ }^{96} \mathrm{Nb}$, processes involved in the ${ }^{96} \mathrm{Mo} \rightarrow{ }^{96} \mathrm{Zr}$ double charge exchange reaction [23]. This is in keeping with the fact that essentially a single $1^{+}$state $(0.69 \mathrm{MeV})$ already implies closure in the present case. To test all these elements, structure, reactions and few-body practitioners have to join efforts, eventually unifying their specific tools into basic physical concepts common to all of the approaches.

Summing up, it is not so much a new, eventually richer and more complete, Hamiltonian to diagonalize ${ }^{1}[30,31]$ that one needs to further nuclear theory, but a new type of nuclear theoretician who computes less and thinks more $^{2}$, and eventually reads the literature, in particular some of the original articles. Like a Renaissance subject, deeply anchored in an exquisite tradition, who was able to combine in a single person the construction worker and the carpenter, the architect and the engineer, the painter and the sculptor, to produce works of great beauty, our theoretical researcher should be equally conversant in structure and reactions, in condensed matter (remarkably closer to the nuclear many-body problem than particle physics) as well as in Standard Model physics, finding him/herself equally at ease in discussing with theoreticians as well as with experimentalists. And the sooner we allow young minds to open up to this scenario, the better. This is also in keeping with the fact that experimental developments, to whom theoreticians are asked to contribute in terms of the physical input, require many years

${ }^{1}$ It is illuminating to quote Pierre-Gilles de Gennes ([30], p. 233) regarding the question asked to him by a student of the prestigious polytechnic School of Paris regarding the answer to a rather simple practical problem: "Mais monsieur, quel est l'Hamiltonien que je dois diagonaliser?" (But sir, which is the Hamiltonian I have to diagonalize?). Let us also mention the work of Transtrum et al. (http://arxiv.org/abs/1501.07668 (2015)) who found that many, if not most high-dimensional models, as well as real processes, are "sloppy", their behavior depending on very few parameters or details. In other words, the distribution of the magnitudes of eigenvalues associated with a variety of models (from radioactive decay to systems biology) and reflecting the relevance of different parameters, fell, according to Transtrum et al., roughly log-linearly, a few parameters (or combinations thereof) tend to be of much greater importance than all others [31]. The above arguments, are to be supplemented with the phenomena of emergent properties, like generalized rigidity in e.g., gauge space $[10,8]$ and pairing rotational bands in nuclei $[28,32-34]$ associated with the phenomenon of spontaneous symmetry breaking restoration. These phenomena are also at the basis of the simplicity of the behavior of complex many-body systems in general, and of the validity of the concepts of collective variables (CV) in nuclei, among which the single-particle motion enters on a par with quadrupole and pairing vibrations and rotations.

${ }^{2}$ Within this context it is sobering to read the following two verses of the poem "Two choruses from "The Rock"" by T.S. Eliott: "Where is the wisdom we have lost in knowledge? where is the knowledge we have lost in information?". 
of planning. A planning which can, at the same time, imply the stop of experimental data-taking, and thus of a partial drought concerning the starting point as well as the nourishment of the theoretical endeavor.

If you want to be an accomplished theorist absorb, as much experimental nuclear physics as you can. Otherwise, you risk to be at the mercy of results you do not understand. Furthermore, you can use this knowledge to play devil's advocate with your most cherished theoretical model. If it passes the proof you will be able to posit that your theory is, at least, not wrong up to that point. Remember that all the great schools of theoretical nuclear physics like Rome and Chicago (Fermi), Copenhagen (Aage Bohr and Ben R. Mottelson), Cornell (Bethe), MIT (Feshbach, Kerman) were strongly connected with experimental activity; activity which got influenced by the work of theorists, but that in turn also influenced in an important way the view of the nucleus held by theorists. Within this context it is worth quoting the answer Niels Bohr gave to Leon Rosenfeld's question on how far the Bohr-Mottelson model was based on first principles, right after Mottelson's report at the 1952 CERN conference. Niels Bohr stated that it appeared difficult to define what one should understand by "first principles" in a world of knowledge where the starting point is empirical evidence of different kinds, not directly combinable.

In fact, it is by playing with the possibility to provide an overall view of the nucleus with its variety of facets and responses to external fields, that you would be able to come close to achieve a consistent physical description of this femtometer system. And to do so start research early, as soon as you feel like doing so, forgetting whether it is ab initio or fundamental. Only whether it is good physics which you really enjoy doing.

Discussions with Gregory Potel, Francesco Cappuzzello, Clementina Agodi, Manuela Cavallaro, Diana Carbone, Arnoldas Deltuva, Francisco Barranco, Enrico Vigezzi, Marcello Baldo and Gianluca Colò are gratefully acknowledged.

Open Access This is an open access article distributed under the terms of the Creative Commons Attribution License (http://creativecommons.org/licenses/by/4.0), which permits unrestricted use, distribution, and reproduction in any medium, provided the original work is properly cited.

\section{References}

1. A. Bohr, B.R. Mottelson, Nuclear Structure, Vol. II (Benjamin, New York, 1975).

2. G. Satchler, Direct Nuclear Reactions (Clarendon Press, Oxford, 1983).

3. C. Mahaux, H. Weidenmüller, Shell model approach to nuclear reactions (North Holland, Amsterdam, 1969).

4. R.A. Broglia, A. Winther, Heavy Ion Reactions (Westview Press, Boulder, CO., 2004).

5. I.J. Thompson, F.M. Nunes, Nuclear Reactions for Astrophysics (Cambridge University Press, Cambridge, 2009).

6. A. Bohr et al., Phys. Rev. 110, 936 (1958).

7. D. Brink, R.A. Broglia, Nuclear Superfluidity (Cambridge University Press, Cambridge, 2005).

8. B.D. Josephson, Phys. Lett. 1, 251 (1962).

9. M.H. Cohen et al., Phys. Rev. Lett. 8, 316 (1962).

10. P.W. Anderson, Special effects in superconductivity, in The Many-Body Problem, edited by E.R. Caianello, Vol. 2 (Academic Press, New York, 1964) p. 113.

11. G. Potel et al., Rep. Prog. Phys. 76, 106301 (2013).

12. R.A. Broglia, D.R. Bes, Phys. Lett. B 69, 129 (1977).

13. F. Cappuzzello et al., Nat. Commun. 6, 6743 (2015).

14. C. Mahaux et al., Phys. Rep. 120, 1 (1985).

15. S.E.A. Orrigo, H. Lenske, Phys. Lett. B 677, 214 (2009).

16. H. Jeppesen et al., Phys. Lett. B 642, 449 (2006).

17. T. Aumann, H. Simon, Nucl. Phys. News 24, 5 (2015).

18. D. Savran et al., Prog. Part. Nucl. Phys. 70, 210 (2013).

19. I. Tanihata et al., Phys. Rev. Lett. 100, 192502 (2008).

20. G. Potel et al., Phys. Rev. Lett. 105, 172502 (2010).

21. V. Rodin, A. Faessler, Phys. Rev. C 80, 041302 (2009).

22. C.J. Guess et al., Phys. Rev. C 83, 064318 (2011).

23. D. Frekers et al., Nucl. Phys. A 916, 219 (2013).

24. H. Ejiri, Phys. Rep. 338, 265 (2000).

25. J. Suhonen, O. Civitarese, Phys. Rep. 300, 123 (1998).

26. F. Cappuzzello et al., J. Phys.: Conf. Ser. 630, 012018 (2015).

27. P.F. Bortignon et al., Giant Resonances (Harwood Academic Publishers, Amsterdam, 1998).

28. D.R. Bès, R.A. Broglia, Nucl. Phys. 80, 289 (1966).

29. D.R. Bès, R.A. Broglia, Phys. Rev. C 3, 2349 (1971).

30. P.G. de Gennes, Les objets fragiles (Plon, Paris, 1994).

31. M. Buchanan, Nat. Phys. 11, 296 (2015).

32. R.A. Broglia et al., Phys. Rep. 335, 1 (2000).

33. G. Potel et al., Phys. Rev. C 87, 054321 (2013).

34. N. Hinohara, W. Nazarewicz, Pairing Nambu-Goldstone modes within nuclear density functional theory, arXiv:1601.00677 (2016). 